\title{
Progress in spin and 3D nucleon structure
}

\author{
Eva-Maria Kabuß ${ }^{* \dagger}$ \\ Institut für Kernphysik \\ D 55099 Universität Mainz E-mail: kabusseuni-mainz.de
}

\begin{abstract}
An overview on studies of the nucleon spin puzzle is given as they are performed in polarised deep-inelastic lepton-nucleon scattering experiments and polarised proton-proton collisions. By now, good knowledge is obtained on the contribution of quark spins to the nucleon spin, while the information about the gluon contribution is still scarce. Currently, the focus is on the study of the orbital angular momentum contribution that is related to the 3-dimensional structure of the nucleon. It is accessible via generalised parton distributions that can be measured in exclusive photon and meson production in lepton scattering experiments. Here, an active experimental and theoretical program is ongoing.
\end{abstract}

XXV International Workshop on Deep-Inelastic Scattering and Related Subjects

3-7 April 2017

University of Birmingham, $U K$

\footnotetext{
*Speaker.

${ }^{\dagger}$ supported by the BMBF
} 


\section{Introduction}

The surprising result of EMC [1] that only a small part of the nucleon spin is carried by quarks resulted in enormous experimental and theoretical activities to perform detailed studies of the nucleon spin. The subsequent measurements confirmed that only one third of the spin of the nucleon is made up by quark spins. It soon became clear that one has to solve the so-called spin puzzle, namely that there can be contributions not only from quark spins but also gluon spins and orbital angular momenta [2]

$$
S_{N}=\frac{1}{2}=\frac{1}{2} \Delta \Sigma+\Delta G+L_{q}+L_{g}
$$

The main tool to study the contribution of quark spins to the nucleon spin is deep inelastic scattering (DIS) of longitudinally polarised charged leptons off longitudinally polarised nucleons. Via scaling violations of the measured spin structure functions $g_{1}\left(x, Q^{2}\right)$ also the contribution of the gluon spin can be studied provided the $Q^{2}$ span of the data is large enough. Here, $x$ is the Bjorken variable and $-Q^{2}$ the squared four-momentum transfer. More insight into the gluon contribution as well as flavour separation of the quark contributions can be obtained from semi-inclusive measurements of DIS (SIDIS) and from proton-proton collisions.

Today these measurements are extended to study not only the longitudinal spin structure but also the 3-dimensional structure of the nucleon by investigating generalised parton distributions (GPDs) $[3,4,5]$.

\section{Experiments}

First measurements of the spin structure were started about 40 years ago at SLAC (E80) and CERN (EMC). When the surprising EMC results became available a series of new experiments were launched at SLAC (first with $30 \mathrm{GeV}$ electrons, later with $50 \mathrm{GeV}$ ), at CERN (SMC, COMPASS), DESY (HERMES) and JLAB (Hall A,B,C). Later the PHENIX and STAR experiments at the polarised proton-proton collider RHIC joined the investigation of the spin structure. Today measurements of GPDs are performed at JLAB and at COMPASS.

\section{Longitudinal nucleon spin structure}

For the investigation of the longitudinal spin structure of the nucleon several processes are used, including inclusive and semi-inclusive measurements of DIS and proton-proton collisions,

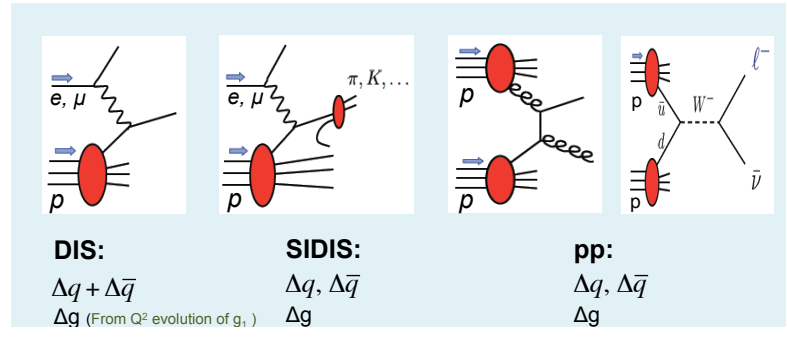

Figure 1: Processes used in the investigation of the spin structure of the nucleon [6]. 
as illustrated in Fig. 1. In DIS, measurements were performed using polarised protons, neutrons and deuterons as targets (see Refs. [7, 8] and references therein). The results for spin structure functions $g_{1}$, which are extracted by measuring longitudinal spin asymmetries, are shown for the proton and the deuteron in Fig. 2. The data include the new final structure function results from COMPASS [8]. These data were also used to extract the non-singlet structure function $g_{1}^{\mathrm{NS}}=$ $g_{1}^{\mathrm{p}}-g_{1}^{\mathrm{n}}$. The first moment of $g_{1}^{\mathrm{NS}}$ at a given value of $Q^{2}$ is connected to the ratio $g_{\mathrm{A}} / g_{\mathrm{V}}$ of the axial and vector coupling constants via the fundamental Bjorken sum rule [9]: $\Gamma_{1}^{\mathrm{NS}}\left(Q^{2}\right)=$ $\int_{0}^{1} g_{1}^{\mathrm{NS}}\left(x, Q^{2}\right) \mathrm{dx}=\frac{1}{6}\left|\frac{\mathrm{g}_{\mathrm{A}}}{\mathrm{g}_{\mathrm{V}}}\right| \mathrm{C}_{1}^{\mathrm{NS}}\left(\mathrm{Q}^{2}\right)$, where $C_{1}^{\mathrm{NS}}\left(Q^{2}\right)$ is the non-singlet coefficient function that can be calculated in perturbative QCD. Using COMPASS data only, a validation of the Bjorken sum rule to an accuracy of about $9 \%$ was obtained [7].
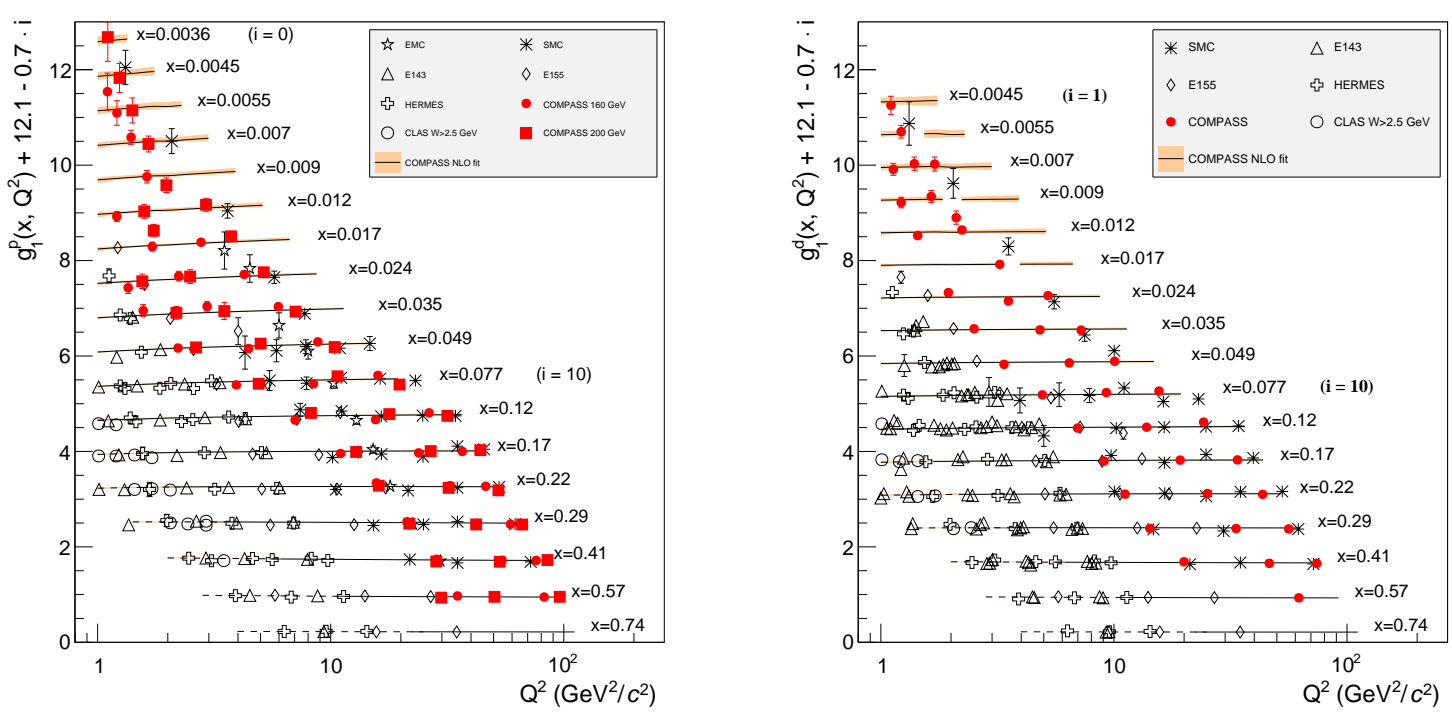

Figure 2: World data on the spin dependent structure function $g_{1}^{\mathrm{p}}$ [7] (left) and $g_{1}^{\mathrm{d}}$ [8] (right) as a function of $Q^{2}$ for various values of $x$. The lines represent the $Q^{2}$ dependence for each value of $x$, as determined from a NLO QCD fit to the world data.

The spin structure functions $g_{1}$ are related to quark and gluon helicity distributions. In LO QCD they are given by

$$
g_{1}\left(x, Q^{2}\right)=\frac{1}{2} \sum_{q} e_{q}^{2} \Delta q\left(x, Q^{2}\right)
$$

with $e_{q}$ the quark charge and $\Delta q=q^{+}-q^{-}$the quark helicity distributions for quarks with their spin parallel (+) or antiparallel (-) to the nucleon spin. The well-known spin independent PDFs are given by $q=q^{+}+q^{-}$. Several groups have performed a next-to-leading order (NLO) QCD analysis of the world data on $g_{1}$ (for a discussion see Ref. [10]). A recent determination of the helicity distributions by the JAM collaboration [11] is shown in Fig. 3. The contribution of all quarks to the nucleon spin is about $30 \%$ with an uncertainty of $10 \%-20 \%$ in all the parametrisations. The $\mathrm{u}$ and d distributions agree quite well in the different analyses. The strange contribution, which is mainly constrained by the assumptions used in the fits, is small and negative with the exception of the DSSV09 extraction [25], which uses in addition semi-inclusive results (discussed below). The 

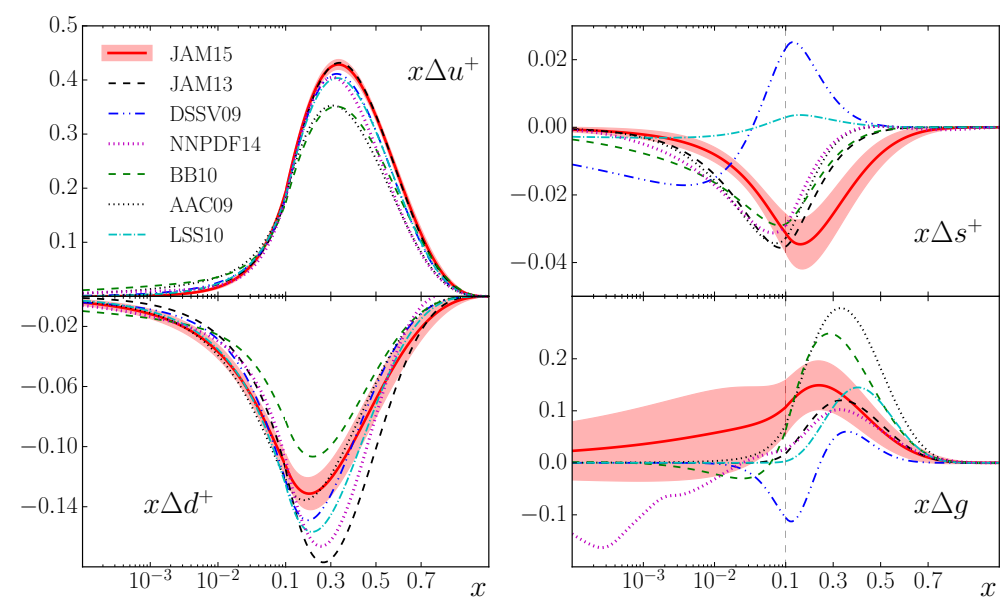

Figure 3: Quark and gluon helicity distributions determined by the JAM collaboration compared to other recent parametrisation (see Ref. [11] and references therein).

gluon distribution is small, but has large uncertainties, so that not even the sign can be determined. The reason is mainly the relatively small $Q^{2}$ span of the available data (see Fig. 2). When data with low invariant mass of the final state hadronic system, $W$, are included in the fits, e.g. the CLAS data, significant higher twist contributions are found.

More $g_{1}$ results are available in specific kinematic regions. The COMPASS collaboration determined asymmetries and spin structure functions for the proton and the deuteron at small values of $x$ down to $5 \cdot 10^{-5}[13,14]$. Due to the fixed target kinematics this implies also small $Q^{2}$. While the deuteron asymmetries are compatible with zero, significant spin effects for the proton were observed for the first time at such low values of $x$. Due to the huge increase of statistics compared to the previous results [12], these data can be used to constrain models of $g_{1}$ at low values of $x$ and $Q^{2}$.

At high $x$ very precise results from JLAB are available; e.g. in HALL A E08-27 a high precision measurement using polarised protons was performed in the resonance region [15]. The main emphasis of the JLAB measurements lies currently on the study of the transition from perturbative QCD to the resonance region to improve the estimates of sum rules, e.g. the generalised GHD sum rule at very low $Q^{2}$. Here, a detailed comparison with predictions from chiral perturbation theory can be done. Figure 4 (right) shows preliminary results from CLAS for the first moment of $g_{1}^{\mathrm{p}}, \Gamma_{1}^{\mathrm{p}}$, (top) and from HALL A E97110 for $\Gamma_{1}^{\mathrm{n}}$ (bottom) [16, 17].

As shown in Fig. 3 the gluon polarisation cannot easily be obtained using inclusive measurements only, as they are dominated by scattering on quarks. To enrich the gluon contribution the photon gluon fusion process (PGF) (see Fig. 5 (left)) can be used in DIS. Here, the PGF process is signalled either by open charm production or by a pair of large transverse momentum hadron (or jets). Figure 5 (right) shows a summary of the existing measurements. The recent COMPASS measurement [18] is the most precise result indicating a small, but positive gluon polarisation around $x=0.1$.

In polarised proton-proton collisions there are three main contributions to the transverse- 

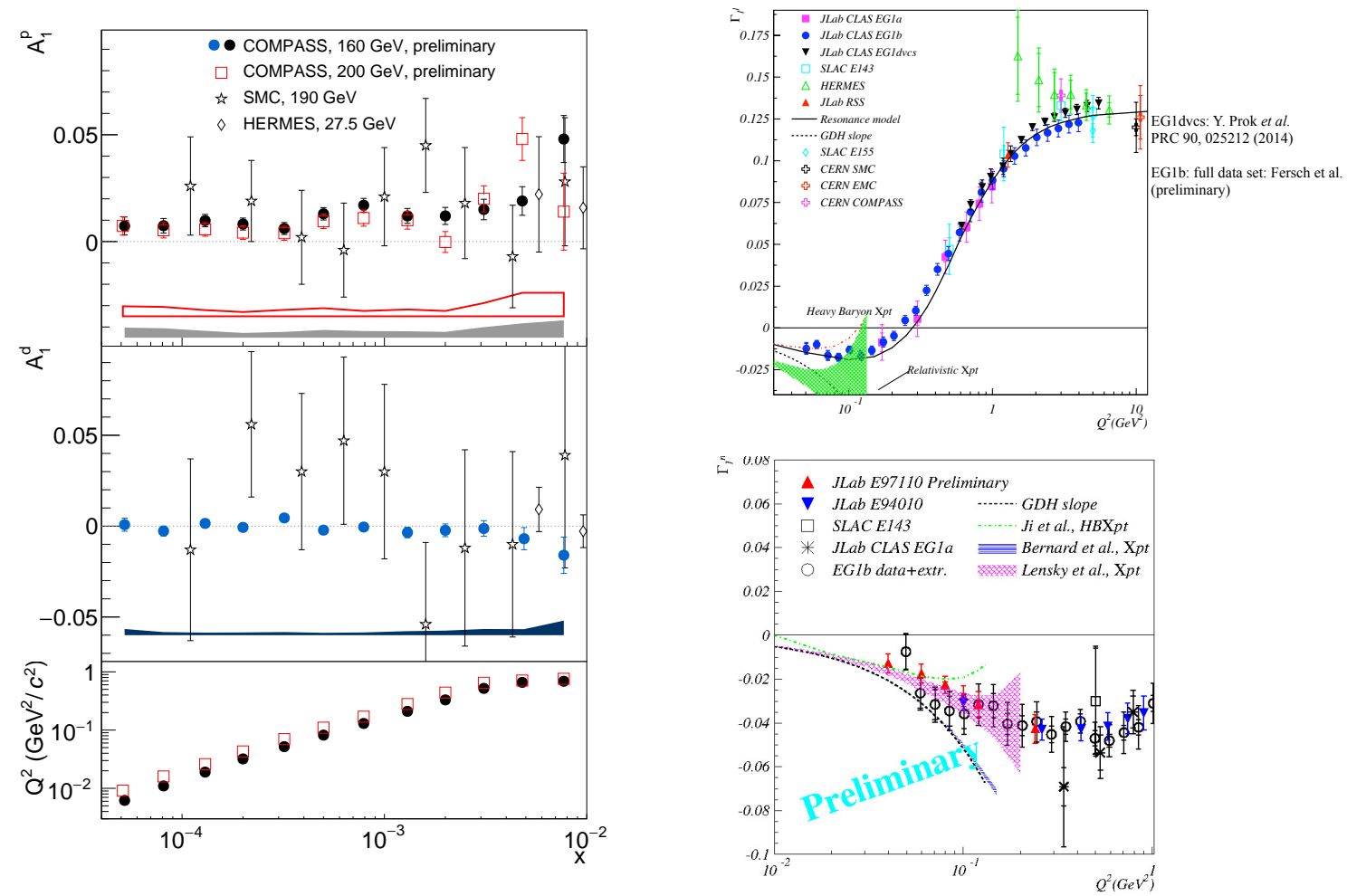

Figure 4: Left: $x$-dependence of the longitudinal double-spin asymmetries $A_{1}^{\mathrm{p}}$ and $A_{1}^{\mathrm{d}}$. The bottom panel shows the average $Q^{2}$ of the COMPASS proton data [13, 14]. Right: Results for $\Gamma_{1}^{\mathrm{p}}$ from CLAS [16] (top) and $\Gamma_{1}^{\mathrm{n}}$ from Hall A E97110 [17] (bottom).
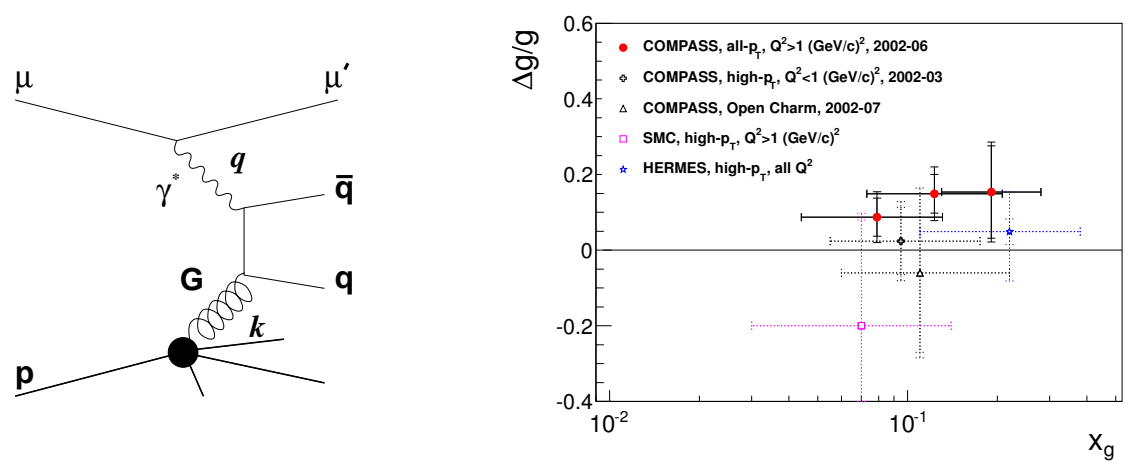

Figure 5: Left: Photon gluon fusion in DIS. Right: World data on direct measurements of the gluon polarisation [18].

momentum dependence of the longitudinal double-spin asymmetry, i.e. quark-quark, quark-gluon and gluon-gluon scattering. The latter two contribute mainly at small and medium transverse momentum $p_{\mathrm{T}}$, yielding access to the gluon polarisation. The quark-gluon process is also sensitive to the sign of the gluon polarisation. In Fig. 6 (left) recent results from PHENIX [19] (top) and STAR [20] (bottom) are presented for the $p_{\mathrm{T}}$ dependence of the longitudinal asymmetry for $\pi^{0}$ and jet production, respectively. The measurements show good agreement with NLO QCD predictions 
using recent polarised PDFs pointing to a small but significant gluon polarisation in the range of $x>0.05$, quite consistent with the LO COMPASS result.
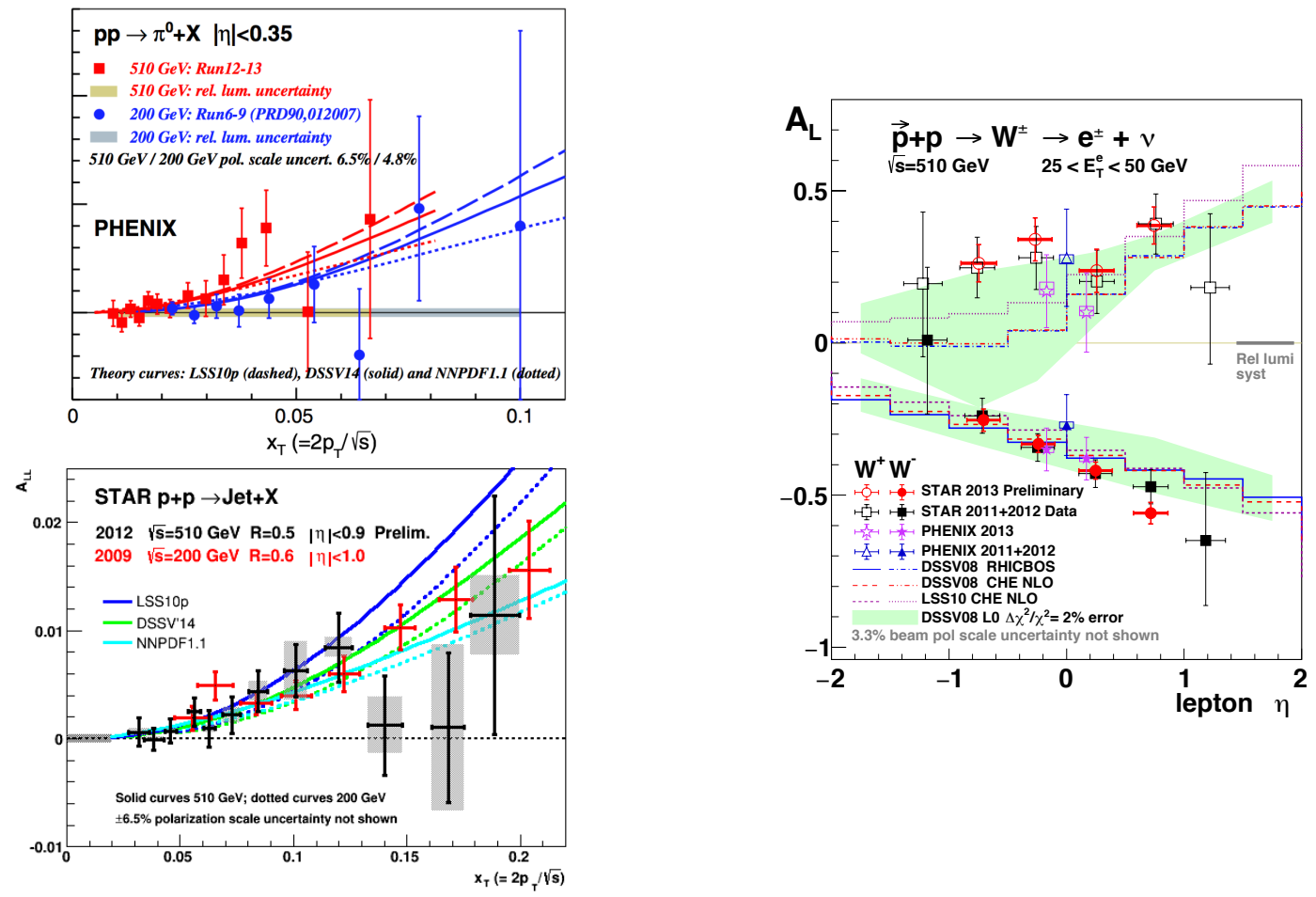

Figure 6: Left: Transverse momentum dependence of the longitudinal asymmetry $A_{\mathrm{LL}}$ for $\pi^{0}$ production from PHENIX [19] (top) and jet production from STAR [20] (bottom). The measurements are compared to predictions using recent polarised PDFs. Right: Dependence of $W^{ \pm}$production asymmetries from PHENIX and STAR on the pseudorapidity $\eta$ in comparison to recent NLO calculation [21].

In proton-proton collisions also weak interaction processes contribute to the measured asymmetries, e.g. $\mathrm{W}^{ \pm}$production at high energies at RHIC. Here, the measurements are sensitive to anti-quark distributions in the proton. Figure 6 (right) shows recent results on $\mathrm{W}$ production from PHENIX and STAR including the new precise STAR result from the 2013 data [21]. The present results point to a sizeable positive contribution from $\Delta \bar{u}$.

The contribution of different quark flavours is studied in SIDIS measurements by COMPASS [23] and HERMES [24]. The sensitivity to quarks and antiquarks occurs through the difference between favoured and unfavoured fragmentation functions (FF) for hadron asymmetries, which are given in LO by

$$
A_{1}^{h}=\frac{\sum_{q} e_{q} \Delta q(x) \int D_{q}^{h}(z) \mathrm{d} z}{\sum_{q} e_{q} q(x) \int D_{q}^{h}(z) \mathrm{d} z} .
$$

Here, $z$ is the ratio of hadron and virtual photon energies and $D_{q}^{h}$ denotes the FF that describes the fragmentation of a quark of flavour $q$ into a hadron of type $h$. While pion FFs are well-known, there are considerable uncertainties for kaon FFs. Thus, $u$ and $d$ quark helicity distribution can be reasonably well constrained by the SIDIS measurements. For strange quarks the resulting helicity 
distribution is usually small and positive (DSSV09 in Fig. 3) in contrast to the one obtained from QCD analyses of inclusive data, but this result changes considerably when another set of kaon FFs is used (see[22]). Therefore, better FFs for kaons are necessary in order to improve the extraction of the strange quark polarisation.

\section{Fragmentation Functions}

Fragmentation functions can be studied in $\mathrm{e}^{+} \mathrm{e}^{-}$collisions and SIDIS measurements (for a summary see Ref. [26]). While the $\mathrm{e}^{+} \mathrm{e}^{-}$data are mainly sensitive to the sum of quark and antiquark FFs, flavour separation can be obtained in SIDIS by measuring multiplicities, which are the ratio of the cross section for hadron production and the DIS cross section. In LO they are related to FFs by

$$
\frac{\mathrm{d} M^{h}}{\mathrm{~d} z}=\frac{\sum_{q} e_{q}^{2} q\left(x, Q^{2}\right) D_{q}^{h}\left(z, Q^{2}\right)}{\sum_{q} e_{q}^{2} q\left(x, Q^{2}\right)} .
$$

Thus, COMPASS [27, 28] and HERMES [29] have measured multiplicities of identified hadrons in multi-dimensional kinematic bins. As an example Fig. 7 shows the precise COMPASS result on the $z$ dependence of multiplicities of $\mathrm{K}^{+}$in bins of $x$ and the relative energy transfer to the nucleon $y$. These new data allow for new extractions of pion and kaon fragmentation functions. A detailed investigation of the experimental results has shown some inconsistencies especially of the COMPASS and HERMES kaon data. Currently a debate is ongoing whether all this data can be analysed in the framework of NLO QCD. New data from COMPASS for kaon multiplicities at very high $z$, as discussed at DIS17[30], will help to shed new light onto these discrepancies.

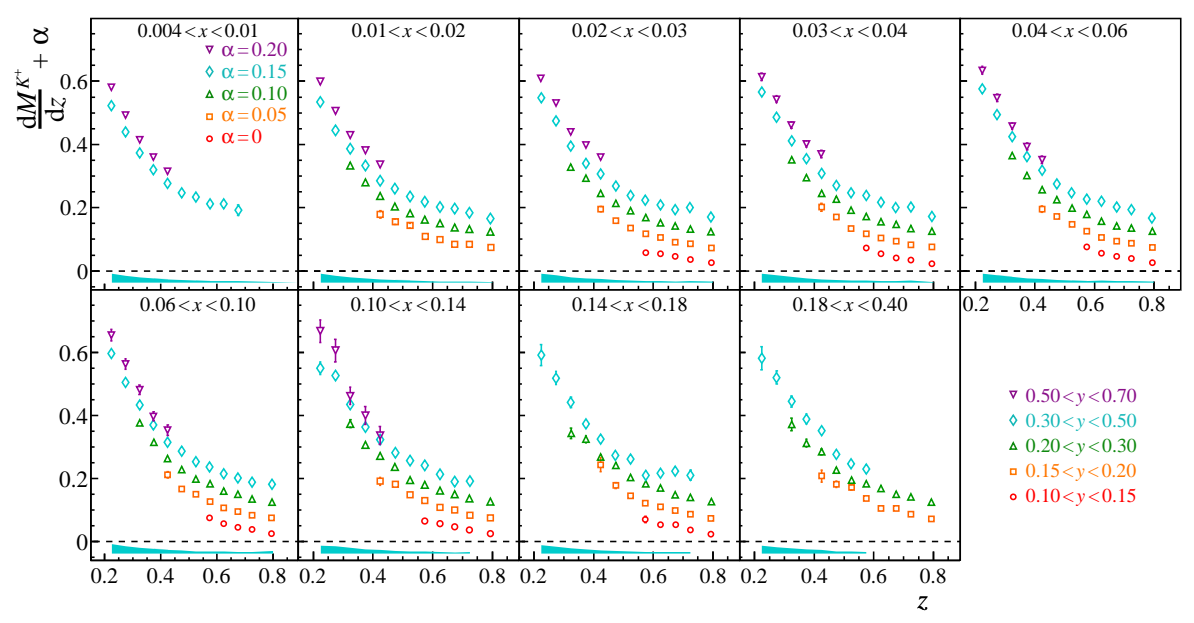

Figure 7: COMPASS results for $\mathrm{K}^{+}$multiplicities in bins of $z, x$ and $y$ [28].

\section{Nucleon structure in $3 D$}

The contributions of quark and gluon spins to the nucleon spin can be investigated in (SI)DIS measurements and proton-proton collisions with longitudinal polarisation. Angular momenta are 
not accessible in such measurements. Access to total angular momenta carried by the nucleon constituents can be addressed by exploiting Ji's sum rule [5]. In this sum rule the angular momenta are related to moments of generalised parton distribution functions (GPD). A GPD can be considered as a momentum-dissected form factor providing information on the transverse localisation of a parton as a function of the fraction it carries of the nucleon longitudinal momentum [3, 4]. Thus one obtains a "3-dimensional picture" of the nucleon, which is often referred to as "nucleon tomography" [31].

These GPDs are accessible via deeply virtual Compton scattering and deeply virtual meson production. They depend on the photon virtuality $Q^{2}$, the total four-momentum transfer squared $t$ between the initial and final nucleon states and two additional variables $x$ and $\xi$ representing the average and half the difference between the initial and final longitudinal momentum fraction of the nucleon carried by the struck parton (see Fig. 8 (left)). In DVCS and DVMP processes $x$ is an internal variable, while the skewness $\xi$ is related to the Bjorken variable $x_{B j}=Q^{2} / 2 M v^{1}$ in the Bjorken limit by $\xi=x_{B j} /\left(2-x_{B j}\right)$.
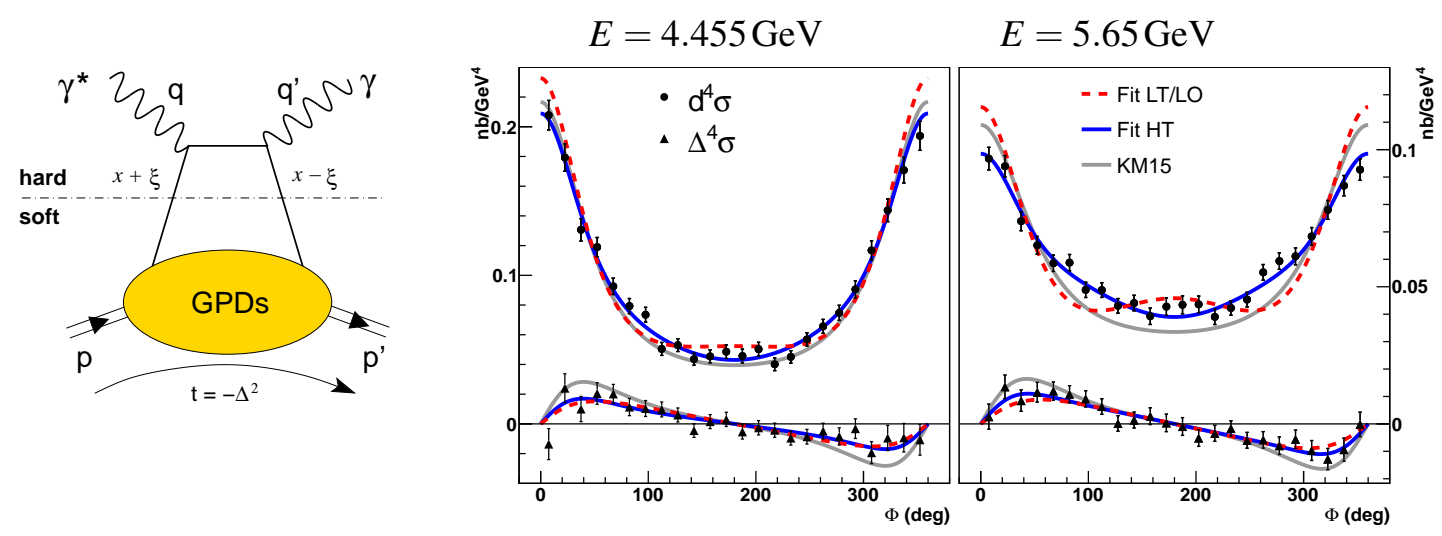

Figure 8: Left: Deeply virtual Compton scattering. Right: Beam helicity $\left(\Delta^{4} \sigma\right)$ and helicity-independent $\left(\mathrm{d}^{4} \sigma\right)$ cross sections at $Q^{2}=1.75 \mathrm{GeV}^{2}, x_{B j}=0.36$ and $t=-0.30 \mathrm{GeV}^{2}$. For the curves see Ref. [32]

A competing process is the Bethe-Heitler $(\mathrm{BH})$ process of elastic lepton-nucleon scattering with a hard Bremsstrahlung photon emitted by the incoming or outgoing lepton. It produces the same final state as DVCS so that both processes interfere at the level of amplitudes. At high beam energies, kinematic domains are accessible where either BH or DVCS dominate. At low $x_{B j}$ almost pure $\mathrm{BH}$ events are recorded, which can be used as an excellent reference yield. The collection of almost pure DVCS events at higher $x_{B j}$ allows for a measurement of the $t$ dependence of the cross section, which is related to the tomographic image of the nucleon. In the intermediate domain the DVCS contribution will be enhanced by the $\mathrm{BH}$ process through their interference. Also for experiments at lower beam energies the DVCS process is mainly accessible via interference with the $\mathrm{BH}$ process.

The global extraction of GPDs, as it is e.g. developed in the PARTONS project [33], needs experimental data on hard exclusive processes in a broad kinematic range. Up to now information in the high energy regime at low $x_{B j}$ was provided by H1 and ZEUS at DESY and by HERMES

\footnotetext{
${ }^{1}$ Note that the variable $x$ in sections $1-4$ is called $x_{B j}$ in section 5 .
} 
and JLAB experiments in the low energy regime at high $x_{B j}$. As an example Fig. 8 (right) shows results from JLab Hall A E07-007 [32]. Here, high statistics data were obtained for the spinindependent cross section as well as for the cross section difference for opposite beam polarisation at several beam energies. A generalised Rosenbluth separation allowed the separation of the BHDVCS interference term. The analysis showed that a leading twist description of the data is not sufficient and higher twist and/or higher order contribution have to be taken into account.

Recently, the COMPASS collaboration has performed a DVCS measurement on protons using oppositely polarised $\mu^{+}$and $\mu^{-}$beams [34]. At high $x_{B j}$ the $t$-dependence of the DVCS cross section is extracted from the cross section sum after integration the azimuthal photon angle and subtraction of the (small) BH contribution. The resulting cross section is shown in Fig. 9 (left). It shows clearly the expected exponential $t$ dependence. The exponential slope $B$ can be related to the transverse size of the nucleon as a function of $x_{B j}$ via $\left\langle r\left(x_{B j}\right)^{2}\right\rangle=2\left\langle B\left(x_{B j}\right)\right\rangle \hbar^{2}$, which is compared to results from ZEUS and $\mathrm{H} 1$ at lower values of $x_{B j}$ in Fig. 9 (right).
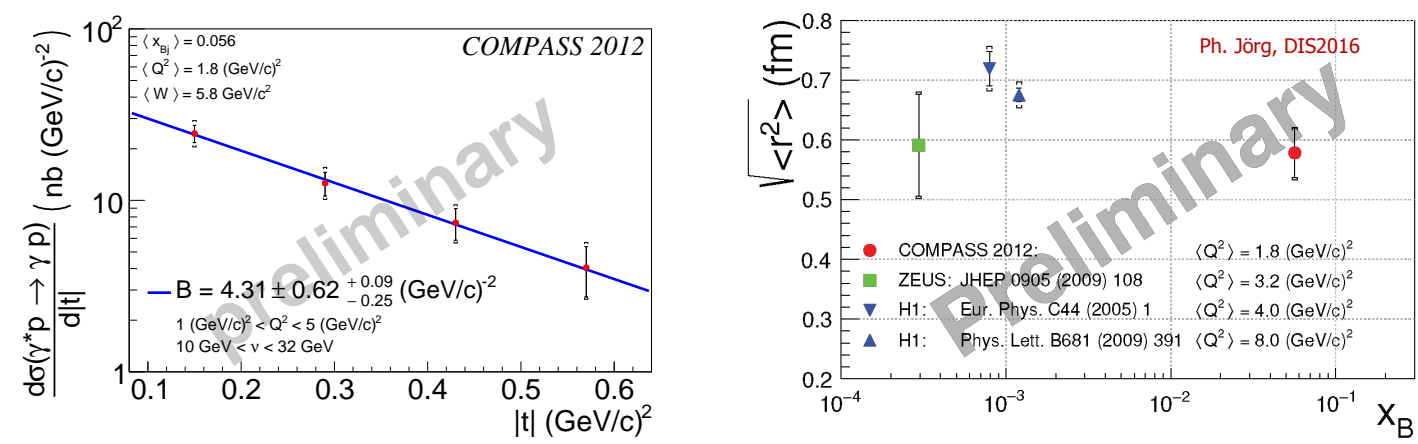

Figure 9: Left: DVCS cross section as function of $|t|$. Right: Comparison of the extracted radius to previous measurements from $\mathrm{H} 1$ and ZEUS. [34]

With the full COMPASS data sample from 2016/17 it will be possible to investigate the $x_{B j}$ dependence of $B$ in the transition region from sea to valence quarks to study a possible shrinkage of the nucleon. The valence region at high $x_{B j}$ was recently investigated by a combined analysis of JLAB and HERMES data (see Ref. [35] and references therein).

\section{Summary and outlook}

After nearly 30 years of measurements the nucleon spin puzzle is only partially solved. More insight e.g. into the gluon contribution will have to wait for the planned electron-ion collider. Meanwhile, the contribution of orbital angular momentum of quarks is investigated using GPDs. Currently an active theoretical and experimental program studying GPDs using exclusive photon or meson production is under the way.

\section{References}

[1] EMC, J. Ashman, et al., Phys. Lett. B 206 (1988) 364; Nucl. Phys. B 328 (1989) 1.

[2] C.A. Aidala, S.D. Bass, D. Hasch and G.K. Mallot, Rev. Mod. Phys. 85 (2013) 655.

[3] D. Mueller et al., Fortsch. Phys. 42 (1994) 101. 
[4] A.V. Radyushkin, Phys. Lett. B 385 (1996) 333; Phys. Rev. D 56 (1997) 5524.

[5] X. Ji, Phys. Rev. Lett. 78 (1997) 610; Phys. Rev. D 55 (1997) 7114.

[6] A. Bazilevsky, Nucleon Helicity Structure: Experimental Overview, talk given at Spin2016 (2016).

[7] COMPASS Collaboration, C. Adolph, et al., Phys. Lett. B 753 (2016) 18.

[8] COMPASS Collaboration, C. Adolph, et al., Phys. Lett. B 769 (2017) 34.

[9] J.D. Bjorken, Phys. Rev. 148 (1966) 1467; Phys. Rev. D 1 (1970) 1376.

[10] E. Nocera, The longitudinal spin structure of the proton: a theory overview, talk given at SPIN2016 (2016).

[11] JAM Collaboration, N. Sato, et al., Phys. Rev. D 93 (2016) 074005.

[12] SMC, B. Adeva, et al., Phys. Rev. D 60 (1999) 072004; erratum ibid. 62 (2000) 079902.

[13] COMPASS Collaboration, M. Aghasyan, et al., arXiv:1710.01014.

[14] COMPASS Collaboration, V.Yu. Alexakhin, et al., Phys. Lett. B 647 (2007) 330.

[15] K. Slifer, Nucleon Spin / JLab program, talk given at JLab workshop on Hadronic Physics with Lepton and Hadron Beams (2017).

[16] CLAS Collaboration, R. Fersch, et al., arXiv:1706.10289.

[17] JLab Hall A Collaboration, J.P. Cheng, private communication.

[18] COMPASS Collaboration, C. Adolph, et el., Eur. Phys. Jour. C 77 (2017) 209.

[19] PHENIX Collaboration, A. Adare, et al., Phys. Rev. D 93 (2016) 011501.

[20] STAR Collaboration, Z.Chang, Inst. J. Mod. Phys. Conf. Ser. 40 (2016) 1660021, arXiv:1512.05400.

[21] STAR Collaboration, S. Gunarathne, Proceedings Spin2016, arXiv:1702.02927.

[22] COMPASS Collaboration, M.G. Alekseev, et al., Phys. Lett. B 680 (2009) 217.

[23] COMPASS Collaboration, M.G. Alekseev, et al., Phys. Lett. B 693 (2010) 227.

[24] HERMES Collaboration, A. Airapetian, et al., Phys. Rev. D 71 (2005) 012003.

[25] D. de Florian, R. Sassot, M. Stratmann, W. Vogelsang, Phys. ReV. D 80 (2009) 034030.

[26] E. Nocera, Fragmentation Functions and their uncertainties, talk given at SPIN2016 (2016).

[27] COMPASS Collaboration, C. Adolph, et el., Phys. Lett. B 764 (2017) 001.

[28] COMPASS Collaboration, C. Adolph, et el., Phys. Lett. B 767 (2017) 133.

[29] HERMES Collaboration, A. Airapetian, et al., Phys. Rev. D 87 (2013) 074029.

[30] COMPASS Collaboration, M. Stolarski, these proceedings.

[31] M. Burkardt, Phys. Rev. D 62 (2000) 071503, erratum-ibid. D 66 (2002) 119903.

[32] JLab Hall A Collaboration, M. Defurne, arXiv:1703.09442.

[33] B. Berthou, et al., arXiv:1512.06174.

[34] COMPASS Collaboration, P. Jörg, PoS DIS2016 (2016) 235.

[35] R. Dupre, M. Guidal, M. Vanderhaeghen, Phys. Rev. G 95 (2017) 011501. 